\title{
TWO-YEAR OUTCOMES OF PULMONARY VEIN ISOLATION AND CAVA-TRICUSPID ISTHMUS RADIOFREQUENCY ABLATION VS PHARMACOLOGICAL ONLY ANTIARRHYTHMIC THERAPY: A SINGLE CENTER EXPERIENCE
}

\author{
Mariia Brynza \\ School of Medicine ${ }^{l}$ \\ Natalia Lisova $\bowtie$ \\ School of Medicine ${ }^{1}$ \\ lesovayanat@gmail.com \\ ${ }^{I}$ V. N. Karazin Kharkiv National University \\ 4, Svobody sq., Kharkiv, Ukraine, 61022
}

$\triangle$ Corresponding author

\begin{abstract}
The benefit of radiofrequency ablation (RFA) in rhythm control in atrial fibrillation (AF) and flutter patients is uncertain, but risk of death, arrhythmia recurrence and other post ablation complications remains high. Existing data on the impact of pulmonary vein isolation and cava-tricuspid isthmus RFA on long-term prognosis of patients with AF and flutter and its advantage over pharmacological antiarrhythmic therapy (AAT) are insufficient and contradictory.

The aim: we sought to evaluate two-year outcomes of pulmonary vein isolation and cava-tricuspid isthmus RFA vs pharmacological only AAT according to a single center experience.

Material and methods: we enrolled 174 patients after pulmonary vein isolation RFA, cava-tricuspid isthmus RFA and their combination and 122 patient who did not undergo RFA and got pharmacological AAT only.

Results: there was no significant difference in mortality between the RFA and AAT only groups ( $5.8 \%$ and $9.0 \%$ respectively) with the same structure of causes of death. The Caplan-Meyer curve analysis demonstrated better survivance $(p=0.031)$ after RFA just during first year of observation. RFA effectiveness in arrhythmia relapse prevention was the highest for cava-tricuspid isthmus RFA procedure and worst - in group of combined pulmonary vein isolation and cava-tricuspid isthmus procedures. RFA showed an advantage over AAT in smaller quantities of non-fatal cardiovascular events $(p<0.001)$ and cardiovascular hospitalizations $(p=0.0026)$.

Conclusions: RFA of pulmonary vein isolation and cava-tricuspid isthmus RFA decrease arrhythmia episodes frequency, risk of non-fatal cardiovascular events and cardiovascular hospitalizations. Timely combined PVI and CTI procedure is associated with worsening of all outcomes.
\end{abstract}

Keywords: radiofrequency ablation, atrial fibrillation, atrial flutter, procedural outcomes.

DOI: $10.21303 / 2504-5679.2022 .002278$

\section{Introduction}

Radiofrequency ablation (RFA) is one of the leading approaches to rhythm control in atrial re-entry arrhythmias. It is a first-choice treatment tactic in symptomatic and high-risk atrial flutter and often prescribed to atrial fibrillation (AF) patients with drug inefficiency or intolerance [1, 2]. RFA - isolation of pulmonary veins for AF and RFA of cava-tricuspid isthmus in right atria for flutter treatment could be used alone or simultaneously to increase the efficiency of a single procedure [3, 4].

Based on previous studies, data on RFA advantage over the pharmacological antiarrhythmic therapy (AAT) are contradictory. Researches show ablation preference in arrhythmia relapse prevention, but the level of post ablation AF and flutter recurrence remains from 40 to $60 \%$ according to various sources $[5,6]$. Most of these cases needed repeated RFA procedures. Data on all-cause mortality levels and general survivance are comparable for RFA and AAT only treatment [7, 8].

Contrariwise, RFA decreases the level of non-fatal cardiovascular events and number of cardiovascular hospitalizations in AF and flutter patients [9, 10], but it is mainly connected to successful ablations. 
There is scarce literature discussion on the impact of pulmonary vein isolation and cavatricuspid isthmus RFA on long-term prognosis of patients with AF and flutter.

Therefore, the aim of this study was to evaluate two-year outcomes of RFA vs pharmacological only AAT according to a single center experience.

\section{Materials and methods}

Study population. 174 patients (mean age $57.2 \pm 9.8$ years, 78 women $(44.8 \%)$ ) with paroxysmal and persistent forms of AF and flutter were enrolled at the department of Ultrasound and Clinical Instrumental Diagnostics and Minimally Invasive Interventions of State Institute «Institute of General and Emergency Surgery named after V. T. Zaitseva NAMS of Ukraine». All patients underwent RFA pulmonary vein isolation and/or cava-tricuspid isthmus from April 2015 to April 2017. We excluded the subjects under 35 years. In order to minimize the influence of high blood pressure and severe heart failure, patients with arterial hypertension III degree and an ejection fraction less than $40 \%$ were excluded. The study population was divided into three groups using type of ablation procedure: RFA pulmonary vein isolation (PVI) $(n=81,47 \%)$, RFA of cava-tricuspid isthmus (CTI) ( $n=34$, $20 \%)$ and RFA both PVI and CTI $(n=59,33 \%) .26(14.9 \%)$ patients underwent re-ablation during study period: 11 (13.6 \%) in PVI group, 7 (20.6\%) in CTI group and 8 (13.6 \%) in PVI and CTI group.

The control group consisted of 122 patients (mean age 58.4 12.4 years, 67 women $(54.9 \%)$ ) with paroxysmal and persistent forms of AF and flutter, who received only pharmacological AAT.

Ethics statement. The study protocol was approved by the institutional review board of the Ethics and Deontology committee in the medical school of V. N. Karazin Kharkiv National University (protocol 12.7 of 15 December 2017). Informed consent was obtained from the patients and our study was performed by the principles expressed in the Declaration of Helsinki.

Electrocardiographic evaluation. To determine presence of arrhythmia, electrocardiogram recording, and evaluation was performed by one experienced physician using an electrocardiography machine (ECG system Cardiolab, Kharkiv, Ukraine) for rest electrocardiography and a Holter monitoring (Holter ECG system Cardiosens K, Kharkiv, Ukraine) for 24-hour electrocardiogram monitoring.

Collection of demographic and medical data. Demographic and medical data including age, gender, disease progression and complications, comorbid conditions and use of medications were collected from recordings and interviews with patients.

Study observation. Patients in groups 1-3 and control group were observed before, 3-5 days, 3, 12 and 24 months after RFA/randomization at the department of Ultrasound and Clinical Instrumental Diagnostics and Minimally Invasive Interventions of SI «Institute of General and Emergency Surgery named after V. T. Zaitseva NAMS of Ukraine».

Definition of all-cause mortality, arrhythmia relapse, non-fatal cardiovascular events and hospitalizations. Survival information and causes of death were obtained from official death certificates. Confirmation of arrhythmia relapse was performed with rest electrocardiography or Holter 24-hour electrocardiogram monitoring. Acute coronary syndrome (ACS) was confirmed by angiography and/or laboratory tests; stroke or transient ischemic attack confirmed by magnetic resonance imaging, laboratory and angiographically confirmed peripheral thrombotic and thromboembolic episodes and major bleedings were defined as a non-fatal cardiovascular event. Hospitalizations due to arrhythmias, coronary artery disease, cerebrovascular disease, hypertension, heart failure or peripheral artery disease were evaluated as cardiovascular and due to all other causes - as non-cardiovascular during study period.

Statistical analysis. SPSS Statistics 23.0 software was used for statistical analysis. Variables following normal distribution were expressed as mean \pm standard deviation or percentage during the follow-up period and analyzed by t-test. For variables following non-normal distribution, data was expressed as median and range and were compared by Wilcoxon test. Non-parametric values were compared by Mann-Whitney U test. Differences of qualitative results were analyzed by chi-square test or Fisher exact test. Survival rates were analyzed by the Kaplan-Meier method and differences between groups were analyzed using the long-rank test. All statistical tests were two-sided, with the threshold of significance set at $p<0.05$ level. 


\section{Results}

There was no difference in age, gender, body mass index (BMI), presence of hypertension and congestive heart failure (CHF) in Groups 1-3 and between RFA and control group (Table 1).

Table 1

The clinical and demographic characteristics of patients that underwent pulmonary vein isolation and cavatricuspid isthmus RFA vs AAT only

\begin{tabular}{|c|c|c|c|c|c|c|}
\hline \multirow{2}{*}{ Variable } & \multicolumn{4}{|c|}{ RFA } & \multirow{2}{*}{ Control group } & \multirow{2}{*}{$p$-value } \\
\hline & In total & PVI & CTI & PVI and CTI & & \\
\hline Age, years & $57.2 \pm 9.8$ & $57.4 \pm 10.2$ & $56.9 \pm 11.6$ & $59.3 \pm 9.7$ & $58.4 \pm 12.4$ & $>0.05$ \\
\hline Gender (female), $\%$ & 44.6 & 48.1 & 41.7 & 45.3 & 51.8 & $>0.05$ \\
\hline BMI, $\mathrm{kg} / \mathrm{m}^{2}$ & $26.5 \pm 4.9$ & $27.4 \pm 5.2$ & $26.7 \pm 4.3$ & $23.1 \pm 6.4$ & $26.8 \pm 5.4$ & $>0.05$ \\
\hline Hypertension, \% & 44.8 & 43.9 & 46.2 & 52.1 & 48.3 & $>0.05$ \\
\hline $\mathrm{CHF}, \%$ & 34.1 & 22.7 & 19.1 & 28.4 & 30.8 & $>0.05$ \\
\hline
\end{tabular}

Note: BMI - body mass index, CHF - chronic heart failure, CTI - cava-tricuspid isthmus, PVI - pulmonary vein isolation, $R F A$ - radiofrequency ablation

The two-year mortality rates in RFA and control groups was $5.8 \%(n=13)$ and $9.0 \%(n=11)$. No statistically significant difference was found between RFA and control in causes of death, with the exception of end-stage heart disease, which caused death only AAT patients. In all groups the most common causes were non-cardiovascular, stroke, acute myocardial infarction (MI) and sudden cardiac death (SCD) (Table 2).

Table 2

Causes of mortality two-years after pulmonary vein isolation and cava-tricuspid isthmus RFA vs AAT only

\begin{tabular}{|c|c|c|c|c|c|c|}
\hline \multirow{2}{*}{ Causes of mortality } & \multicolumn{4}{|c|}{ RFA } & \multirow{2}{*}{$\begin{array}{l}\text { Control } \\
\text { group }\end{array}$} & \multirow{2}{*}{ p-value } \\
\hline & In total & PVI & CTI & PVI and CTI & & \\
\hline Non-cardiovascular, \% (n) & $4.0(7)$ & $4.9(4)$ & $2.9(1)$ & $5.1(3)$ & $2.5(3)$ & $>0.05$ \\
\hline Stroke, \% (n) & $2.3(4)$ & $1.2(1)$ & 0 & $5.1(3)$ & $4.9(6)$ & $>0.05$ \\
\hline Acute MI, \% (n) & $0.6(1)$ & 0 & 0 & $1.7(1)$ & $1,6(2)$ & $>0.05$ \\
\hline $\mathrm{SCD}, \%(n)$ & $0.6(1)$ & $1.2(1)$ & 0 & 0 & $0.8(1)$ & $>0.05$ \\
\hline End-stage heart disease, $\%(n)$ & 0 & 0 & 0 & 0 & $3.3(4)$ & $>0.05$ \\
\hline
\end{tabular}

Note: CTI - cava-tricuspid isthmus, PVI - pulmonary vein isolation, RFA - radiofrequency ablation, SCD - sudden cardiac death

Result of Kaplan-Meier curve analysis is displayed in Fig. 1, illustrating the cumulative two-year mortality among the RFA and control (Fig. 1, A), and among different RFA procedures and control (Fig. 1, B). The results suggested that the risk of death decreased for RFA in the first year $(p=0.031)$ and was comparable in two-year observation $(p>0.05)$, there was no significant difference between types of ablation $(p>0.05)$.

$\mathrm{AF}$ and/or flutter relapse frequency in first 3 months for all RFA procedures was not evaluated as a blind period (Table 3). RFA effectiveness was the highest for CTI procedure, where arrhythmia episodes were observed two times less often in the first year $(p<0.001)$ and remained stable; and worst - in group of combined PVI and CTI procedures, where the level of AF and and/or flutter relapse gradually increased till 2 years after RFA $(p=0.019)$.

Percentage of non-fatal cardiovascular events was significantly lower $(p<0.001)$ in patients after RFA in comparison with AAT only patients (Fig. 2). In the first 3 months only one event (myocardial infarction) was observed after RFA in PVI group, while in AAT only group $4.9 \%$ of patients had different events. Upon further observation, this difference increased (5.1\% RFA vs $14.8 \%$ AAT only after first year; 6.2-10.2\% RFA vs $20.5 \%$ AAT only after second year). 

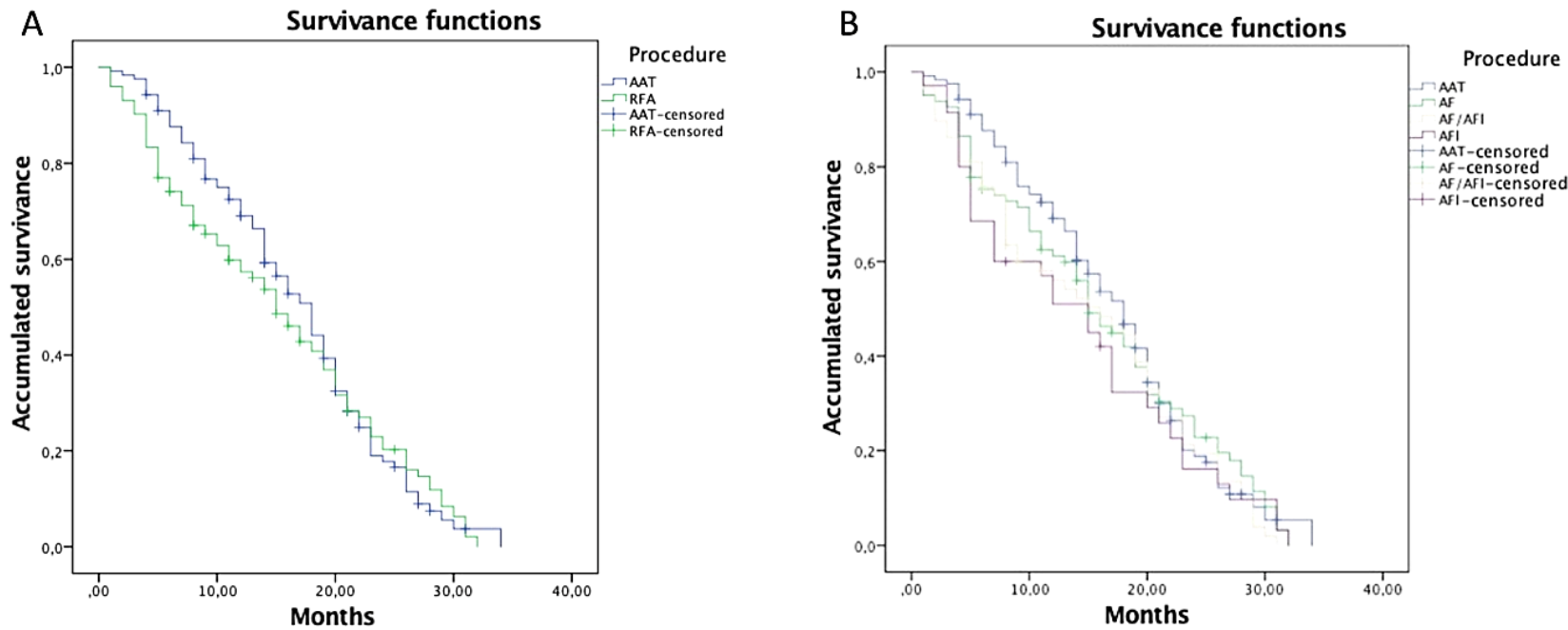

Fig. 1. Result of Kaplan-Meier curve analysis is displayed

Table 3

Arrhythmia relapse frequency after pulmonary vein isolation and cava-tricuspid isthmus RFA

\begin{tabular}{ccccc}
\hline \multirow{2}{*}{ RFA } & \multicolumn{4}{c}{ Relapse in period } \\
\cline { 2 - 5 } & $\mathbf{3}$ months, \% (n) & $\mathbf{1 2}$ months, \% (n) & $\mathbf{2 4}$ months, \% (n) & p-value \\
\hline In total & $27.0(47)$ & $23.0(40)$ & $24.1(42)$ & $>0.05$ \\
PVI & $25.9(21)$ & $21.0(17)$ & $19.0(16)$ & $>0.05$ \\
CTI & $35.3(12)$ & $20.6(7)^{*}$ & $11.8(4)^{* *}$ & $* * *<0.001$ \\
PVI and CTI & $23.7(14)$ & $28.8(17)$ & $35.6(21)^{*}$ & $* 0.019$
\end{tabular}

Note: CTI-cava-tricuspid isthmus, PVI-pulmonary vein isolation, RFA-radiofrequency ablation

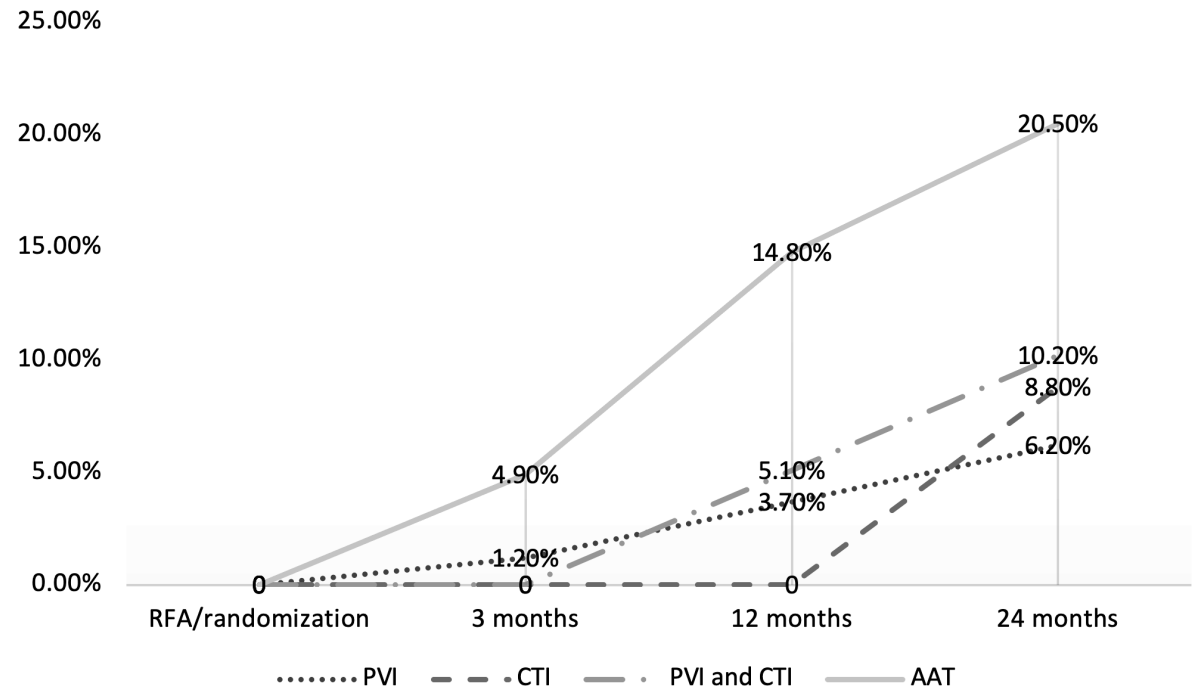

Fig. 2. Comparison of the percentage of non-fatal cardiovascular events in patients after RFA with patients treated with AAT alone

Rate of cardiovascular hospitalizations was significantly lower in RFA group in comparison with AAT only group $(p=0.0026)$ regardless of the type of procedure. Hospitalizations for non-cardiovascular causes comparable in most study groups (Table 4). 
Table 4

Hospitalization rates two-year after RFA vs AAT only

\begin{tabular}{|c|c|c|c|c|c|c|}
\hline \multirow{2}{*}{ Hospitalizations } & \multicolumn{4}{|c|}{ RFA } & \multirow{2}{*}{ Control group } & \multirow{2}{*}{ p-value } \\
\hline & In total & PVI & CTI & PVI and CTI & & \\
\hline Cardiovascular, \% (n) & $28.2(49)$ & $22.2(18)$ & $26.5(9)$ & $37.3(22)$ & $55.7(68)^{*}$ & $* 0.0026$ \\
\hline Non-cardiovascular, \% (n) & $9.2(16)$ & $6.2(5)$ & $11.8(4)$ & $11.9(7)$ & $10.7(13)$ & $p>0.05$ \\
\hline
\end{tabular}

Note: CTI-cava-tricuspid isthmus, PVI-pulmonary vein isolation, RFA-radiofrequency ablation

\section{Discussion}

Results from this study demonstrated no significant difference in all-cause mortality level between RFA and AAT only strategies ( $8.6 \%$ vs $9.0 \%$ ) for rhythm control in patients with AF within a two-year period, which is close to the CABANA study report $[4,11](8.0 \%$ vs. $9.2 \%)$, although it included older patients or patients with risk factors for stroke. At the same time, mortality structure did not differ in patients after ablation and AAT only: the leading causes were non-cardiovascular and stroke, patients also died due to myocardial infarction and ventricular arrhythmias. Furthermore, our results demonstrated that death as a result of chronic condition, such as end-stage heart failure was observed just in patients who did not undergo RFA. Conversely, data of big systematic review and meta-analysis [12] revealed that ablation was associated with lower risk of all-cause mortality vs medical management only; at the same time the authors indicated that evidence is limited to heart failure and left ventricle systolic dysfunction. Regarding our study, outcomes were evaluated in patients with preserved systolic function, which was not reflected previously. Some papers connected with systolic heart failure suggested a connection between catheter ablation and fewer death from worsening heart failure in comparison with standard conventional therapy [5]; it was noted that the benefit becomes augmented as follow up is extended from 1 to 5 years [13]. The current study showed advantage of RFA vs AAT in patients' survival only in the first year of observation, which subsequently did not reach a significant level.

Furthermore, data of mortality in patients after cava-tricuspid isthmus RFA are very contradictory too, and unlike AF, very limited. Significantly lower mortality levels (2.9\%) in patient with atrial flutter after RFA was present in our study, which was comparable to meta-analysis of 18 primary studies with 22 treatment arms and 1323 patients [3], where all-cause mortality level reach just $0.6 \%$. On the other hand, the observational study that was based on data from Danish nationwide health databases were reported higher mortality risk after atrial flutter ablation procedures compared with patient undergoing AF ablation [14].

The current study has demonstrated significant prevalence of ablation vs. AAT only in prophylaxis of AF and/or flutter recurrence for all RFA procedures: pulmonary vein isolation, cava-tricuspid isthmus ablation and their combination. Ablation efficiency from $64.4 \%$ to $85.3 \%$ in our research is comparable to current guidelines [1] and data of recent studies [6]. The least effective treatment was shown for combined PVI and CTI procedure: we observed increasing of arrhythmia relapse levels in study period after RFA; there was no significant change in PVI group and decrease in arrhythmia episodes frequency during study in CTI group. Partly, we associate this with highest re-ablation level: every fifth patient after CTI RFA for two years underwent PVI RFA. Data of CTI RFA effectiveness are very contradictory: number of papers described frequency of AF recurrence in nearly $30 \%$ and flutter recurrence $-15 \%$ of patients [7], more successful flutter ablation in $85 \%$ of cases was associated just with the left ventricle procedure [9], and [14] reported even higher renewed arrhythmia levels after flutter RFA in comparison with AF. In first three months post-ablation, usually evaluated as a blind period, arrhythmia episodes were registers in $23.7-35.3 \%$ of patients. It was noted that patients with AF or flutter recurrence in the second and third month more often had arrhythmia relapses in future observations, especially in patients with high arrhythmic load. A similar correlation was described previously, where the authors suggested to consider 23 days or about 3 weeks (rather than 3 months) to be an appropriate cut-off for the blanking period [15].

Based on current analysis, RFA shows it advantage over AAT only strategy in non-fatal cardiovascular events. Moreover, this difference gradually increased during investigation and reached 
6.2-10.2\% after ablation vs $20.5 \%$ in control group, almost half of the patients from all this groups suffered strokes, which became the leading cardiovascular cause of death and the leading non-fatal event. The benefit of RFA vs. AAT only in stroke prevention was confirmed in UK registry [10] and was non-reproduceable in large US national registry [11] and other studies [4]. Additionally, it should be noted that the effectiveness of combined procedure PVI and CTI RFA, including for events prevention, remains uncertain $[4,16]$. Our finding is at variance with it, we demonstrated more rare non-fatal cardiovascular events in PVI and CTI group in comparison with AAT only, but it was the highest of all RFA procedures.

In all groups of patients, analyzing frequency of hospitalizations after RFA vs AAT only strategies we found that the rate of cardiovascular hospitalizations was significantly lower in post-ablation patients. This was also indicated in previous papers analyzing the advantage of RFA over AAT only in cardiovascular hospitalizations $[4,17]$. The most often re-admission $(37.3 \%)$ we observed after combined PVI and CTI procedures.

Finally, the benefit of CTI RFA for typical atrial flutter in our study is reflected very ambiguous in another works. Some papers reported surprisingly high rates of cardiovascular events and hospitalizations [8, 18], and some - similar to ours had better outcomes after CTI procedure in comparison with AF ablation [19, 20].

Study limitations. The study evaluated the data of 174 patients after RFA of pulmonary vein isolation, RFA of the tricuspid isthmus and their combination and 122 patients who did not undergo RFA and received only pharmacological AAT. This is a sufficient reference sample. The level of quality of life was not discussed.

Prospects for further research. It looks important to do further investigations to evaluate predictors of RFA effectiveness, especially for atrial flutter or flutter with AF patients.

\section{Conclusions}

Based on two-year observation, the PVI and CTI RFA outcomes are promising. All ablation procedures decrease arrhythmia frequency and duration, risk of non-fatal cardiovascular events and cardiovascular hospitalizations. Timely combined PVI and CTI procedure is associated with worsening of all outcomes.

There was no significant difference in mortality between the RFA and AAT only groups $(5.8 \%$ and $9.0 \%$ respectively) with the same structure of causes of death. The Caplan-Meyer curve analysis demonstrated better survivance $(p=0.031)$ after RFA just during first year of observation. RFA effectiveness in arrhythmia relapse prevention was the highest for cava-tricuspid isthmus RFA procedure and worst - in group of combined pulmonary vein isolation and cava-tricuspid isthmus procedures. RFA showed an advantage over AAT in smaller quantities of non-fatal cardiovascular events $(p<0.001)$ and cardiovascular hospitalizations $(p=0.0026)$.

\section{Conflict of interests}

The authors declare that they have no conflicts of interest.

\section{Financing}

The study was performed without financial support.

\section{References}

[1] Kirchhof, P., Benussi, S., Kotecha, D., Ahlsson, A., Atar, D., Casadei, B. et. al. (2016). 2016 ESC Guidelines for the management of atrial fibrillation developed in collaboration with EACTS. European Heart Journal, 37 (38), 2893-2962. doi: http:// doi.org/10.1093/eurheartj/ehw210

[2] Yi, F., Hou, W., Zhou, C., Yin, Y., Lu, S., Duan, C. et. al. (2019). Radiofrequency Ablation Versus Antiarrhythmic Drug Therapy for Atrial Fibrillation. Journal of Cardiovascular Pharmacology, 73 (4), 241-247. doi: http://doi.org/10.1097/ fjc.0000000000000654

[3] Spector, P., Reynolds, M. R., Calkins, H., Sondhi, M., Xu, Y., Martin, A. et. al. (2009). Meta-analysis of ablation of atrial flutter and supraventricular tachycardiaThe American Journal of Cardiology, 104 (5), 671-677. doi: http://doi.org/10.1016/ j.amjcard.2009.04.040 
[4] Packer, D. L., Mark, D. B., Robb, R. A., Monahan, K. H., Bahnson, T. D. et. al. (2019). Effect of Catheter Ablation vs Antiarrhythmic Drug Therapy on Mortality, Stroke, Bleeding, and Cardiac Arrest Among Patients With Atrial Fibrillation. JAMA, 321 (13), 1261-1274. doi: http://doi.org/10.1001/jama.2019.0693

[5] Shah, S. R., Moosa, P. G., Fatima, M., Ochani, R. K., Shahnawaz, W., Jangda, M. A., Shah, S. A. (2018). Atrial fibrillation and heart failure- results of the CASTLE-AF trial. Journal of Community Hospital Internal Medicine Perspectives, 8 (4), $208-210$. doi: http://doi.org/10.1080/20009666.2018.1495979

[6] Tsiachris, D., Giannopoulos, G., Deftereos, S., Kossyvakis, C., Tsioufis, C., Siasos, G. et. al. (2019). Biomarkers Determining Prognosis of Atrial Fibrillation Ablation. Current Medicinal Chemistry, 26 (5), 925-937. doi: http://doi.org/10.2174/0929867325666180320122930

[7] Warchoł, I., Bińkowski, B. J., Kucejko, T., Sobiczewska, J., Lubiński, A. (2019). A Retrospective Study of Atrial Fibrillation Following Cavotricuspid Isthmus Ablation for Atrial Flutter. Medical Science Monitor, 25, 3316-3320. doi: http://doi.org/10.12659/msm.912918

[8] Steinbeck, G., Sinner, M. F., Lutz, M., Müller-Nurasyid, M., Kääb, S., Reinecke, H. (2018). Incidence of complications related to catheter ablation of atrial fibrillation and atrial flutter: a nationwide in-hospital analysis of administrative data for Germany in 2014. European Heart Journal, 39 (45), 4020-4029. doi: http://doi.org/10.1093/eurheartj/ehy452

[9] Giehm-Reese, M., Lukac, P., Kristiansen, S. B., Jensen, H. K., Gerdes, C., Kristensen, J. (2019). Outcome after catheter ablation for left atrial flutter. Scandinavian Cardiovascular Journal, 53 (3), 133-140. doi: http://doi.org/10.1080/14017431.2019.1612086

[10] Jarman, J. W., Hunter, T. D., Hussain, W., March, J. L., Wong, T., Markides, V. (2017). Stroke rates before and after ablation of atrial fibrillation and in propensity-matched controls in the UK. Pragmatic and Observational Research, 8, 107-118. doi: http:// doi.org/10.2147/por.s134781

[11] Freeman, J. V., Shrader, P., Pieper, K. S., Allen, L. A., Chan, P. S. et. al. (2019). Outcomes and Anticoagulation Use After Catheter Ablation for Atrial Fibrillation. Circulation: Arrhythmia and Electrophysiology, 12 (12). doi: http://doi.org/10.1161/circep.119.007612

[12] Barra, S., Baran, J., Narayanan, K., Boveda, S., Fynn, S., Heck, P. et. al. (2018). Association of catheter ablation for atrial fibrillation with mortality and stroke: A systematic review and meta-analysis. International Journal of Cardiology, 266, 136-142. doi: http://doi.org/10.1016/j.ijcard.2018.03.068

[13] Bunch, T. J., May, H. T., Afshar, K., Alharethi, R., Day, J. D. (2019). Mechanisms of Improved Mortality Following Ablation: Does Ablation Restore Beta-Blocker Benefit in Atrial Fibrillation/Heart Failure. Cardiology Clinics, 37 (2), 177-183. doi: http:// doi.org/10.1016/j.ccl.2019.01.011

[14] Skjøth, F., Vadmann, H., Hjortshøj, S. P., Riahi, S., Lip, G. Y. H., Larsen, T. B. (2018). Disease progression after ablation for atrial flutter compared with atrial fibrillation: A nationwide cohort study. International Journal of Clinical Practice, 72 (11), e13258. doi: http://doi.org/10.1111/ijcp.13258

[15] Alipour, P., Azizi, Z., Pirbaglou, M., Ritvo, P., Pantano, A., Verma, A., Khaykin, Y. (2017). Defining Blanking Period Post-Pulmonary Vein Antrum Isolation. JACC: Clinical Electrophysiology, 3 (6), 568-576. doi: http://doi.org/10.1016/j.jacep.2017.01.006

[16] Rodgers, M., McKenna, C., Palmer, S., Chambers, D., Van Hout, S., Golder, S. et. al. (2008). Curative catheter ablation in atrial fibrillation and typical atrial flutter: systematic review and economic evaluation. Health Technology Assessment, 12 (34). doi: http://oi.org/10.3310/hta12340

[17] Brynza, M. (2021). Quality of life prognosis in patients two years after radiofrequency ablation of atrial fibrillation. Theoretical and empirical scientific research: concept and trends, 2. doi: http://doi.org/10.36074/logos-28.05.2021.v2.40

[18] Evans, J. M., Withers, K. L., Lencioni, M., Carolan-Rees, G., Wood, K. A., Patrick, H., Griffith, M. (2019). Quality of life benefits from arrhythmia ablation: A longitudinal study using the C-CAP questionnaire and EQ5D. Pacing and Clinical Electrophysiology, 42 (6), 705-711. doi: http://doi.org/10.1111/pace.13675

[19] Gottlieb, L. A., Dekker, L. R. C., Coronel, R. (2021). The Blinding Period Following Ablation Therapy for Atrial Fibrillation: Proarrhythmic and Antiarrhythmic Pathophysiological Mechanisms. JACC: Clinical Electrophysiology, 7 (3), $416-430$. doi: http://doi.org/10.1016/j.jacep.2021.01.011

[20] Shantha, G., Alyesh, D., Ghanbari, H., Yokokawa, M., Saeed, M., Cunnane, R. et. al. (2019). Antiarrhythmic drug therapy and all-cause mortality after catheter ablation of atrial fibrillation: A propensity-matched analysis. Heart Rhythm, 16 (9), 1368-1373. doi: http://doi.org/10.1016/j.hrthm.2019.06.007

How to cite: Brynza, M., Lisova, N. (2022). Two-year outcomes of pulmonary vein isolation and cava-tricuspid isthmus radiofrequency ablation vs pharmacological only antiarrhythmic therapy: a single center experience. EUREKA: Health Sciences, 1, 10-16. doi: http://doi.org/10.21303/2504-5679.2022.002278 